\title{
Optimizing Enterprise IT Infrastructure through Virtual Server Consolidation
}

\author{
Qiyang Chen and Rubin Xin \\ Montclair State University, Montclair, NJ, USA \\ chenq@mail.montclair.edu xingr@mail.montclair.edu
}

\begin{abstract}
As computing power and storage needs increase rapidly, companies are looking for ways to reduce costs and improve overall return on investment. Although this is not market responsibility, a better understanding of this knowledge would greatly help managers of the companies that are wishing to enhance their current information technology infrastructure.

The purpose of this study is to review issues of virtual server consolidation. Factors contributing to the both the positives and negatives associated with the implementation of virtual server consolidation will be evaluated in order to provide increased awareness of this emerging technology. Practical knowledge is discussed to help information technology managers explore this emerging technology for their enterprise information technology infrastructure.
\end{abstract}

Keywords: virtual server

\section{Introduction}

In recent years, there has been a rapid growth in server computing. From large enterprise data centers to modest sized companies, hardware costs continue to climb even though the retail costs of hardware have fallen. In addition to upfront costs associated with hardware, additional expenses are added. It is not uncommon for these companies to have many servers spread around for various departments. These servers are often purchased over a period of time by different decision makers from various vendors.

As businesses have grown and expanded their information technology needs, more new servers have been to purchased and installed. Since the initial purchase price of an Intel-based server has fallen, many purchasing decisions have been delegated to the department level. Since the separation of purchasing decisions have been established, it is not unusual to see a mixture of different vendors and architectural differences among these servers.

This influx in physical server resources causes companies to consider ways to reduce the management, overhead, and complexity involved in maintaining existing server-based networks. Companies that recognize these symptoms in their information technology architecture are also looking for economic efficiencies that will reduce costs while at the same time continuing, or sur-

Material published as part of these proceedings, either on-line or in passing, the level of productivity in print, is copyrighted by Informing Science. Permission to make digital or paper copy of part or all of these works for personal or classroom use is granted without fee provided that the copies are not made or distributed for profit or commercial advantage AND that copies 1) bear this notice in full and 2) give the full citation on the first page. It is permissible to abstract these works so long as credit is given. To copy in all other cases or to republish or to post on a server or to redistribute to lists requires specific permission from the publisher at Publisher@InformingScience.org 


\section{Issues of Server Sprawl}

Beginning in the late 1980's changes in the information technology market added distributed computing and the client/server architecture as guiding principles for advancement in information technology. As businesses grew and their information technology demands increased, companies had to acquire and deploy solutions to keep pace but at the same time keep costs down. Distributed servers are an economical way to meet these needs. However, as the amount of servers increased so did the overhead associated with maintaining these servers increased. Information technology infrastructures became complicated, distributed, and harder to manage and support, causing "server sprawl." A technology used to combat server sprawl is server consolidation. Server consolidation is the process of merging the uses of two or more servers to a single server. Recently, organizations have increased their need beyond consolidation of two or more servers onto one server. In order to address this issue server consolidation through virtualization becomes the newest emerging technology.

In an enterprise data center server sprawl contributes significantly to the costs of running an effective information technology infrastructure. This effect multiplies with company growth. This infrastructure problem results in a business problem for the entire organization. Some applications conflicted with each other and had to run on separate servers. Furthermore, the performance of shared applications and databases housed at the central data center have been degenerating (Aharoni, 2003). The IT departments also need the ability to manage applications without adding headcount while maintaining the company's decentralized style.

A traditional approach often would not satisfy the following service requirements:

- Maintaining file and application servers at each office while centralizing databases, messaging and directory services,

- Replicating critical application databases to each office,

- Opting for individual use of servers for each application to avoid conflicts, and

- Increasing network bandwidth to handle database and directory synchronization traffic.

These requirements imply managing many servers across dedicated WAN links, which would incur high recurring costs without even taking disaster-recovery capabilities into account. It also would continue to stretch a small staff too thin, requiring frequent travel to all locations (Dubie, 2004).

\section{New Approach in Consolidation}

The answer to these infrastructure issues (server sprawl and distributed computing) is virtual server consolidation. Unlike the server consolidation that took place in the late 1990's, consolidating two or more applications built on separate servers and merging onto one server, virtual server consolidation is more in depth. Consolidation is not new in the enterprise data center. Companies are already benefiting from the ability to merge several applications on one operating system. These applications all use the available resources from the physical hardware as well as the server operating systems. Since these applications have to share memory, hard disk space, and the central processor, many applications still cannot be merged onto on physical server with one operating system. Server virtualization (or virtual server consolidation) enables multiple virtual operating systems to run on a single physical machine, yet remain logically distinct with consistent hardware profiles. The "host" operating system creates an illusion of partitioned hardware by executing multiple "guest" operating systems (Burry \& Nelson, 2004). 


\section{Virtual Consolidation}

Server virtualization is the masking of server resources (including the identity of individual physical servers, processors, and operating systems) from server users, freeing users from having to be aware of these resources while optimizing resource sharing. Server virtualization is intended to spare the user from having to understand and manage resources while increasing overall resource utilization and the ability to expand later. Server virtualization is closely identified with the concept of the virtual machine, the assignment of a copy of an operating system and the appearance of a full set of storage and other resources to each user.

In the mainframe computer world with IBM's VM (now called z/VM) operating system, each of many simultaneous users of the mainframe (physical server) is given apparent control of the entire computer with their own copy of an operating system and all other needed resources. In many businesses today, smaller Wintel-powered computer servers and more powerful Unix servers, all originally designed for one user at a time, are increasingly being used to serve many user requests. For these servers, several recent hardware and software approaches to server virtualization have been provided, including the use of multiple processors in each server and multiple partitions sharing a processor, with each partition having its own copy of the operating system (Kovar, 2003). As with the mainframe, server virtualization screens the user or application program from having to know which processor and partition is doing the service.

The advantages of server virtualization also includes: the ability to have all developers share the same machine while developing and testing new programs; in some cases, the ability to support legacy applications (which run on an older version of an operating system) while newer applications run on the latest operating system level on the same server; and the ability to simulate distributed network applications on the same physical server.

Server virtualization is sometimes described as a better approach than server consolidation, the effort to reduce the number of servers handling a workload, because, while using existing resources more efficiently, it offers the possibility of future growth. Server virtualization can be viewed as part of an overall virtualization trend that includes storage virtualization and network virtualization, although these are sometimes just included as part of server virtualization. Server virtualization is seen as a likely requirement for utility computing in which computer processing power is seen as a utility that clients can pay for only as needed, and also in grid computing in which an array of computer processing resources, often in a distributed network, are used for a single application.

\section{Advantages of Consolidation}

Server consolidation offers the following advantages:

- Reduced total cost of ownership

- Improved efficiency

- Improved service levels

- Infrastructure for growth

Reduced total cost of ownership: There are several costs involved in deploying and maintaining an information technology infrastructure, such as hardware, staff support, operational procedures, and hidden costs. Virtual server consolidation reduces the number of physical servers thereby cutting hardware costs, not only for the server unit, but also a reduction in peripherals needed. These additional peripherals, such as network adapters, switches, cabling and backup devices are expensive items that contribute to the cost of hardware infrastructure (Bort, 2004). Consolidation will allow consistent system administration with a goal geared toward a reduction in downtime. 
This will require less attention from the support staff, allowing the staff to use their time for other matters. Less physical hardware means less operational expense such as power consumption and floor space. Hidden costs are those associated with wasted resources such as disorganization, reliability, and time management.

It has been acknowledged that one of the key drivers of change in the enterprise data center in next few years is the need to control costs (Bort, 2004). Between 60 percent and 80 percent of IT budgets is tied up in managing the assets a data center. In next few years we will also see a continued focus on shrinking the number of vendor partners as a way for enterprise data centers to cut costs (Dubie, 2004). Vendor consolidation eases the burden of managing data centers while giving enterprises fewer throats to choke. But there are others that feel it could decrease innovation (Segall, 2005). Vendor reduction for physical servers will allow information technology departments to reduce hardware support contracts since the majority support contracts are based on a per server basis.

Improved efficiency: Many physical servers do not use $100 \%$ of their resource capability (Bort, 2003). With consolidation, server usage can be increased to a satisfactory level since the virtual hardware can be tailored to the operating system. Rather than purchasing different levels of hardware from different vendors, a level of efficiency increases when that demand is available with a few clicks.

Since a pool of server operating systems are running on the same physical hardware, data center automation and monitoring tools will take advantage of the availability, monitoring, and utilization capabilities from one vendor. As compared with operating physical servers from different vendors, separate hardware and automation tools were needed to manage. Decreasing these tools to one allows for greater concentration to one application (Patel, 2002).

Improved service levels: With a consolidated infrastructure it is much easier to implement a 24/7 support operation. Response time is greater than in a distributed environment and data is more accessible while maintaining security. The latest servers with new technology focused on virtual server platforms have a higher level of stability, redundancy, and dependability, which increases the IT level of services (Segall, 2005).

Infrastructure for growth: Consolidation offers scalability and flexibility to add or remove servers as needed. Increasing computing resources can be implemented through the virtualization application. In the traditional physical server scenario increasing the amount of CPU required adding an additional CPU (if the physical server was capable) or purchasing new equipment and migrating the operating system and its applications. If the need arrives where the host physical hardware needs to be upgraded, migration of the "virtualized" server is a snap. By encapsulating a specific virtual machine (an application and its operating system needs), any application can be nearly instantly ported to another available server (Wang, 2002).

\section{Potential Problems of Consolidation}

Server consolidation may also have the following potential problems:

- Central point of failure

- Lack of vendor application support

- Employee perspective

Central Point of Failure: When consolidating multiple physical servers onto one physical hardware server you are "putting all your eggs in one basket.” When examining server consolidation, IT managers should be aware of the limitations associated with this approach. One important issue is the server becomes a central point of failure, a problem with it could knock all users off- 
line. In comparison, the single server approach impacts only those users working with a particular application. Increasing the fault tolerance and redundancy will increase costs associated with the additional hardware, management, and overhead.

Lack of vendor application support: Many vendors may not provide product support of their applications if they are not running on a physical server. This is a major issue since product support plays a key role in making sure service levels agreements are met. For instance, although Microsoft has released their own virtual server software support policies, the company sets for Windows products already affected VMware users. Users of VMware, which is the current market leader for server vitualization, indicated that those policies cost them time and convenience (Aharoni, 2003). Microsoft won't support its applications or Windows operating systems that are running in the VMware environment. It requires that any problem be replicated on a physical server before the support is offered. This means that companies deploying server virtualization via VWmare will have to keep spare physical servers to meet Microsoft's requirements for reproducing issues on a hardware platform or forgo their product support plans (Brandel, 2003).

Employee perspective: When pointing fingers about the decrease of IT jobs, workers and industry watchers often blame offshore outsourcing. But in fact, new technology could share the blame. AMR Research, Forrester Research and Gartner separately report that server consolidation; network, server and storage virtualization; and product road maps toward utility computing ultimately will lessen the need to add entry-level positions in enterprise data centers (Dubie, 2004). And, in some cases, emerging IT implementations could result in layoffs. A reduction in physical servers may be interpreted as a reduction in support staff. The idea, although may not be true, can cause issues with an employee's perspective on his/her job with the company. Support staff personal may also feel a lack of control. IT support teams or individuals that previously had their own server may object to the loss of control and flexibility created by server consolidation.

\section{Virtualization in Non-Production Environments}

Virtual server consolidation is an attractive alternative to server sprawl. Server virtualization is an action item on nearly every IT agenda these days. Gartner predicts that by the end of 2005, 25\% of the Fortune 1000 will use partitioning - a key virtualization technology - for their Windows server deployments. And by 2008, it is estimated that companies that don't leverage virtualization technologies will spend 25\% more for their Intel servers and 15\% more for RISC servers, including hardware, software, labor and space (Thibodeau, 2004 ).

Still, users might have a hard time drawing a bead on the various server virtualization architectures - what the strategies are, which are best for their environment, which will accommodate virtualization needs for storage and network resources, and how much the architectures lock them into the vendors' products. Companies that are skeptic about moving to virtual server consolidation can still benefit by first deploying this architecture in their non-production environments (Bort, 2003).

As discussed, there are advantages and disadvantages of virtual server consolidation. However, in a non-production (testing, development, or disaster recovery) scenario the advantages far out weight the disadvantages. Since non-production departments can sustain downtime, "putting all your eggs in one basket" does not have the high level impact, as would a production environment. This also stands for application and vendor support since other support options can be researched without time constraints.

Organizations that cannot afford a duplication of their production environment cannot effectively test patches and new deployments in a production scenario. They are forced to either forgo deployment of critical server patches and updates or deploy the updates to their production systems and hope they do not cause a conflict, or worse, a disaster. This may become a major issue for 
some organization. Organizations need not only faced cost cutting issues in their production environment, but also low-cost development and test environments for internal customers. Thus, organizations will bill customers for the costs of both development and production servers, but the customers are willing to pay for production costs only. Because development environments were virtually non-existent, companies that are trying sociability testing and managing upgrades on live systems may not only suffer from disruptive operation but also face increasing administrative workload and risk (Thibodeau, 2004).

Deploying virtual server consolidation in a non-production environment not only reduces the costs associated with testing, development, or disaster recovery testing, but also gives companies a chance to "test virtual server consolidation." Although many companies are turning to this emerging technology to benefit their information technology infrastructure, many other companies remain hesitant due to fear. As the technology grows and gains increased consumer confidence, it can be expected that more companies start their quality testing on virtual server consolidation.

\section{Conclusion}

The growth of distributed computing has lead to an increase in server sprawl in information technology infrastructures. Virtual server consolidation enables the deployment of multiple, secure, independent virtual operating system environment on standard physical servers. The purpose of this deployment scenario is to provide an efficient and high performance platform to combat server sprawl. As companies increase server sprawl, information technology managers should adopt virtual server consolidation as an alternative solution.

As organizations are struggling with the management physical servers, it is necessary to evaluate the advantages and disadvantages of virtual server consolidation in their production environments. For companies needing to improve their non-production environments, virtual server consolidation's advantages far out-shine the disadvantages. Testing and deployment environments are ideal since less risk is involved with deploying virtual server consolidation in a nonproduction environment.

As an emerging technology, virtual server consolidation will continue to grow. As many leading vendors, such as IBM, HP, Microsoft and Intel add more research and development investment toward virtual server consolidation, more developments will be geared toward this direction.

Server virtualization technologies will also bring mainframe-like workload consolidation and flexibility to reduced instruction set computer. In addition, re-provisioning technologies will expand the boundaries of RISC- and Intel-based workloads, enabling them to be dynamically "rightsized" to larger or smaller servers when needed. The changes and offerings will be incremental, but they will build to result in significant cost and labor savings throughout the period, as well as increase flexibility to deploy new virtual servers quickly, change resource allocations to workloads quickly, and dispose of virtual servers quickly. Virtualization should become an ongoing effort and a part of the server strategy for every enterprise.

\section{References}

Aharoni, E. (2003, September). A vision of togetherness. Journal of Network and Systems Management, 10 (3).

Bort, J. (2003). Server virtualization: Controlling server sprawl. IEEE Communications Magazine, 41 (2), 126-133.

Bort, J. (2004, February 16). Making the case for the new data center. Network World. 
Optimizing Enterprise IT Infrastructure

Brandel, M. (2003). Wired over server virtualizations. IEEE/ACM Transactions on Networking, 2 (3), 202210.

Burry, C. M. \& Nelson, C. (2004). Plan on server virtualization. Computer Communications, 24 (15), 15251539.

Dubie, D. (2004, May 1). Data center staffing. Network World.

Kovar, J. F. (2003). Reinventing the corporate data center. Computer Communications, 21 (13), 1123-1129.

Patel, B. (2002). Server consolidation through utility computing. Computer Networks and ISDN Systems, 24 (2), 131-144.

Segall, E. (2005, January 7). Improving economics of blades through server consolidation and virtualization” Network World.

Thibodeau, P. (2004, July 12). Microsoft plays price card to grab virtualization share. Network World.

Wang, S. (2002). Virtually embracing success. ACM Transactions on Networking, 7 (6), 906-916.

\section{Biographies}

Dr. Qiyang Chen is an associate professor at the Information and Decision Science Department, Montclair State University, New Jersey.

Dr. Rubin Xin is an assistant professor at the Information and Decision Science Department, Montclair State University, New Jersey. 